Relations industrielles

Industrial Relations

\title{
Administration du travail et conflits collectifs, par Jean-Paul Bachy, Sceaux, CRESST, 1979, 164 pp.
}

\section{Dimitri Weiss}

Volume 35, numéro 2, 1980

URI : https://id.erudit.org/iderudit/029077ar

DOI : https://doi.org/10.7202/029077ar

Aller au sommaire du numéro

Éditeur(s)

Département des relations industrielles de l'Université Laval

ISSN

0034-379X (imprimé)

1703-8138 (numérique)

Découvrir la revue

Citer ce compte rendu

Weiss, D. (1980). Compte rendu de [Administration du travail et conflits collectifs, par Jean-Paul Bachy, Sceaux, CRESST, 1979, 164 pp.] Relations industrielles / Industrial Relations, 35(2), 349-350.

https://doi.org/10.7202/029077ar

Tous droits réservés @ C Département des relations industrielles de l'Universite Laval, 1980
Ce document est protégé par la loi sur le droit d'auteur. L’utilisation des services d'Érudit (y compris la reproduction) est assujettie à sa politique d'utilisation que vous pouvez consulter en ligne.

https://apropos.erudit.org/fr/usagers/politique-dutilisation/ 
application dans des expériences américaines et françaises, Dechêne dépouille une cinquantaine d'ouvrages et offre pour chacun un résumé succint mais substantiel. Ces résumés et commentaires sont présentés de manière à ce que le lecteur puisse rapidement se faire une opinion sur l'ouvrage en question. Cette seconde partie du travail de Dechêne présente une valeur pédagogique certaine.

En résumé, cette publication peut être conseillée à une personne non familière avec le sujet et désireuse d'obtenir une vue d'ensemble synthétique et critique de l'évolution des modes d'organisation du travail. Elle peut constituer une source de documentation féconde pour des étudiants. On n'y trouvera cependant pas de points de vue nouveaux ou de développement originaux.

\section{Alain VINET}

Université Laval

\section{Administration du travail et conflits collec- tifs, par Jean-Paul Bachy, Sceaux, CRESST, 1979, 164 pp.}

La littérature française des relations industrielles, passablement déficitaire sur le thème de l'intervention des pouvoirs publics dans les conflits collectifs du travail, s'enrichit d'une étude d'autant plus utile qu'en dépassant les frontières héxagonales, elle se place sur un terrain international et comparé.

En effet, après un chapitre sur «l'État et les conflits" où ne sont pas rares les références à l'Amérique du Nord, Bachy nous offre, dans un second chapitre, quatre cas étrangers: la Belgique, le Danemark, l'Allemagne fédérale et la Grande Bretagne, qui représentent, au-delà du sujet stricto sensu autant d'occasions d'introduction aux systèmes nationaux de relations du travail des pays respectifs, dont les singularités (et, aussi, implicitement, les convergences) sont bien mis en évidence.

Le troisième chapitre, le plus important en volume, est, naturellement, consacré à la
France. La disproportion entre le poids de la loi et celui de la négociation collective est, dès le début, abordé, et ceci prend toute son importance en ce début de décennie, quand de nouveaux équilibres se font jour dans le jeu des acteurs sociaux et que les employeurs réclament - et oeuvrent pour - des négociations «individualisées» au niveau de l'entreprise, au détriment de dispositions légales et contractuelles au plan des branches d'activité.

Les mécanismes de conciliation (conventionnelle et réglementaire), d'arbitrage et de médiation sont expliqués et évalués, et leur faible efficacité relevée. Le rôle actif - et insubstituable - de l'inspection du travail et l'élargissement de ces compétences sont ensuite mis en relief, de même que les obstacles que l'auteur a décelés au cours de son enquête, tels que le faible nombre de ses membres, qu'il voudrait voir dotés d'une autonomie supérieure, et - de là - les freins apportés parfois par l'autorité supérieure.

Bachy n'a pas manqué, dans sa recherche dont l'intérêt ne faiblit jamais, ni de demander l'avis des partenaires sociaux, ni poser le problème, il y a quelques années très débattu, de l'objectivité d'un corps fortement (et diversement) syndiqué, ni, in fine, de nous livrer quelques renseignements sur la sélection et la formation des inspecteurs.

Des considérations comparées clôturent ce document, rapport de recherche, pertinent, simple - je veux dire dépourvu de toute sophistication académique (mais, également, d'une table des matières...), écrit dans un langage clair et accessible, qui dit l'essentiel sans prétention d'exhaustivité. Un ouvrage nécessaire et sérieux qui s'ajoute à celui que l'auteur avait publié, il y a deux ans, sur Les jeunes et la société nouvelle (CRESST, 1977) et qui remplit, très méritoirement, un vide.

Des cinq pays qu'il a traités, il manque, toutefois, à mes yeux, le sixième. Ceci d'autant plus que l'Italie nous avait offert, il y a déjà plusieurs années, un remarquable ouvrage - de juriste, mais écrit dans une perspective de relations industrielles -, que je 
tiens pour un livre de référence, particulièrement en Europe latine, sur la médiation des pouvoirs publics dans les conflits collectifs du travail: La mediazione dei publici poteri nei conflitti collettive di lavoro (Bologna, Il Mulino, 1972). L'auteur, Bruno Veneziani, dont j'ai déjà eu l'occasion de parler dans ces colonnes à propos de deux autres volumes collectifs parus en 1978 (voir Relations industrielles, vol. 34, no. 1, 1979, pp. 205-206), est également le signataire d'une contribution sur le même sujet au 26e volume de l'Encyclopédie du Droit: "Mediazione nelle controversie collettive di laboro" (Enciclopedia del diritto, Milano, Giuffrè Editore, 1976).

\section{Dimitri WEISS}

Institut d'Administration des Entreprises, Paris

Les nouvelles formes d'organisation du travail, vol. 2, Genève, Bureau international du travail, 1979, $161 \mathrm{pp}$.

Dans un premier volume (Les nouvelles formes d'organisation du travail, 1979), le Bureau international du travail a déjà publié cinq monographies sur les nouvelles formes d'organisation du travail au Danemark, en Norvège et en Suède, en République fédérale d'Allemagne, en France, au Royaume-Uni et aux États-Unis. Dans ce deuxième volume, le BIT s'intéresse à des expériences tentées en Italie, en Inde, en République démocratique allemande et en URSS.

Bien que l'avant-propos comprenne un avertissement concernant le caractère disparate des analyses regroupées dans ce second volume, le lecteur est néanmoins assuré d'y trouver une trame, un commun dénominateur. En effet, tous les auteurs manifestent d'une façon ou d'une autre une préoccupation envers des considérations théoriques: fondements des systèmes d'organisation du travail, évolution du marché de l'emploi, répercussions du progrès technique, principes d'organisation etc. De plus, pour chaque pays, on s'attarde à décrire le système d'organisation du travail en vigueur ainsi que la démarche jugée la plus appropriée pour con- tribuer à son amélioration. Enfin, dans tous les cas, des exemples concrets sont présentés.

Mais là s'arrêtent les points communs. Certaines monographies ont été rédigées par des chercheurs indépendants tandis que d'autres l'ont été par des spécialistes à l'emploi d'organismes of ficiels. Les opinions émises à l'égard de l'organisation du travail varient largement et se situent de façon différente par rapport au système socio-économique des pays concernés. En regroupant ces monographies, l'objectif du BIT n'était pas de prendre position sur les opinions exprimées mais de montrer «comment les chercheurs ou les spécialistes des organismes of ficiels envisagent le problème dans différents pays». Au lecteur de juger. Le choix des expériences est varié et comprend aussi bien des groupes semi-autonomes dans la métallurgie italienne que l'application du système scientifique d'organisation du travail dans l'industrie textile soviétique.

Cette publication du BIT comprend également un article de Anthony Hopwood consacré à l'évaluation de l'intérêt économique des nouvelles formes d'organisation du travail. À la suite de son analyse, l'auteur conclut que les normes visant à améliorer la qualité de la vie au travail, bien qu'elles soient parfois coûteuses, «peuvent avoir une incidence positive sur les résultats économiques généraux des entreprises». À partir d'exemples, il discute des facteurs agissant sur les résultats économiques des mesures d'amélioration de la qualité de la vie au travail. Cependant, ce type d'évaluation n'est pas sans poser de délicats problèmes de mesure au point d'exiger souvent une remise en cause de la façon traditionnelle d'envisager les performances et les résultats de l'entreprise.

Alain VINET

Université Laval

Administration industrielle et générale, par Henri Fayol, édition présentée par P. Morin, Paris, Dunod, 1979, 156 pp.

Les spécialistes et observateurs des relations industrielles sont familiarisés avec ce 